\title{
Antifibrotic action of interleukin-1 receptor antagonist in lapine monoarticular arthritis
}

\author{
Jo Lewthwaite, Simon Blake, Robert C Thompson, Timothy E Hardingham, \\ Brian Henderson
}

Division of

Biochemistry,

Kennedy Institute of

Rheumatology,

Bute Gardens,

London W6 7DW,

United Kingdom

J Lewthwaite

T E Hardingham

Maxillofacial Surgery

Research Unit,

Eastman Dental

Institute for Oral

Health Care Sciences,

University of London,

256 Gray's Inn Road,

London WC1X 8LD,

United Kingdom

$S$ Blake

B Henderson

Synergen Inc,

1885 33rd Street,

Boulder,

Colorado 80301, USA

R C Thompson

Correspondence to:

Professor Brian Henderson

Accepted for publication

13 February 1995

\begin{abstract}
Objective-To determine if the administration of interleukin-1 receptor antagonist (IL-1ra) to animals with established antigen induced arthritis had any beneficial effects on the synovitis and cartilage destruction.

Methods-Antigen induced arthritis was induced in New Zealand White rabbits, and after two weeks IL-1ra was administered every six hours over a 72 hour period. Animals were then killed and joint tissues examined for the degree of synovitis, synovial fibrosis, and cartilage damage.

Results-The response of the arthritis to the treatment was minor in terms of joint swelling, leucocyte accumulation, or cartilage proteoglycan loss. However, the synovial fibrosis was not only halted by administration of IL-1 ra, but reversed. The inflamed synovial linings of IL-1ra treated animals showed a significant loss of synovial collagen content and a reappearance of the synovial fat spaces which are prominent in the normal synovial lining.

Conclusion-This study shows that IL-1ra has potent antifibrotic activity and suggests the use of this agent for the reversal of the fibroproliferative process which is so important in the pathology of rheumatoid arthritis.
\end{abstract}

(Ann Rheum Dis 1995; 54: 591-596)

The synovitis associated with rheumatoid arthritis is also accompanied by a profound proliferative and fibrotic response which can clearly be seen when the joint is opened at surgery. The rheumatoid synovial lining becomes enlarged, growing over and into the articular cartilage, and normally forms a mass of villous processes which protrude into the joint space. This fibroproliferative response is one of the complex processes that leads to the destruction of articular cartilage and subchondral bone in this disease. ${ }^{1}$

Cytokines and growth factors are likely to be involved in this synovial fibroproliferative response, but there are a number of problems in defining their precise role. Most human rheumatoid synovium available for study comes from patients at the end stage of disease, by which time the fibroproliferative response is likely to be much less active. It is only in recent years that agents specifically inhibiting cytokine synthesis or function have become available for clinical evaluation.

It is possible to test hypotheses concerning the role of cytokines and growth factors in synovial fibroproliferation by use of animal models of rheumatoid arthritis. One model that has been used extensively and which mimics the synovial fibroproliferative response of rheumatoid arthritis is antigen induced arthritis in the rabbit. ${ }^{2-4}$ Induction of arthritis produces a swift proliferative response in many of the resident cell populations ${ }^{25}$ which is associated with the accumulation of leucocytes in the synovial subintima. ${ }^{3}$ A striking finding is the rapid increase in collagen content of the inflamed synovial lining. ${ }^{6}$

Two members of the interleukin (IL)-1 family, IL- $1 \alpha$ and IL-1 $\beta$, are potent proinflammatory molecules with the capacity to stimulate mesenchymal cells, including synovial fibroblasts, to proliferate and produce increased levels of connective tissue macromolecules. ${ }^{7}$ Thus it is possible that, by blocking the activity of IL-1, one could inhibit this fibroproliferative response in the inflamed synovial lining. The specific blockade of IL-1 is now possible by utilising the third member of the IL-1 family-interleukin-1 receptor antagonist. This protein has the capacity to bind to the type I IL-1 receptor without triggering an agonist response, and thus functions as a receptor antagonist. ${ }^{78}$ It can therefore be used to identify pathology caused specifically by this cytokine.

Previously, we have shown that the intravenous administration of IL-1 ra is effective in inhibiting the synovitis and cartilage proteoglycan loss caused by the intra-articular injection of IL-1. ${ }^{9}$ In a subsequent study, we found that IL-1 ra had no inhibitory effect on swelling, leucocyte accumulation or cartilage proteoglycan loss when administered to rabbits with acute antigen induced arthritis. ${ }^{10}$ These results suggest that $\mathrm{IL}-1$ is not a major mediator of the induction phase of this experimental lesion.

In terms of the management of rheumatoid arthritis, it is obviously of more interest to determine what effect inhibitors such as IL-1 ra have on the established disease. Therefore, in the present study we have examined the effects of administering IL-1 ra to rabbits in which disease had been induced two weeks earlier. At this stage the joints are chronically inflamed and there are marked fibroproliferative changes in the inflamed synovial lining. ${ }^{2} 6$ 


\section{Materials and methods}

INDUCTION OF ANTIGEN INDUCED ARTHRITIS Seventeen female New Zealand White rabbits (initial body weight $2 \cdot 2-2 \cdot 5 \mathrm{~kg}$ ) were immunised with $1 \mathrm{ml}$ of Freund's complete adjuvant (FCA) (Sigma) containing $5 \mathrm{mg}$ ovalbumin (Sigma) in mutiple intradermal sites. Animals were reimmunised 14 days later using the same procedure. Five days after the second immunisation, arthritis was induced in the left knee by the injection of $1 \mathrm{ml}$ of a sterile solution of ovalbumin in saline $(5 \mathrm{mg} / \mathrm{ml})$ into the joint cavity. As a control, the contralateral joint was injected with $1 \mathrm{ml}$ of sterile saline. All procedures were performed in strict accordance with current Home Office regulations.

\section{ASSESSMENT OF ARTHRITIS}

The development of arthritis was determined by measuring joint swelling using a pair of callipers according to a standardised procedure. Knee joints were flexed at a $45^{\circ}$ angle and the width across the middle of the joint measured in both the inflamed and the contralateral control joints. The degree of swelling was obtained by subtracting the latter value from the former.

Animals were killed by administration of an overdose of pentobarbitone. Blood was collected by cardiac puncture just before death and was placed in heparinised tubes and centrifuged at $400 \mathrm{~g}$ for 10 minutes at $4^{\circ} \mathrm{C}$. The plasma was stored at $-20^{\circ} \mathrm{C}$ before assay of IL-1ra. To collect the synovial fluid and associated infiltrating leucocytes, $1.5 \mathrm{ml}$ of sterile saline was injected into the joint cavity, the joints were gently flexed to mix the contents, and were then opened and the joint fluid carefully collected into a syringe. This fluid is referred to as the joint wash. Cells present in this joint wash were pelleted by centrifugation in an Eppendorf centrifuge for 30 seconds. The volume of the cell free joint fluid was measured and divided into aliquots and immediately frozen on solid carbon dioxide and stored at $-70^{\circ} \mathrm{C}$. Leucocytes were resuspended in saline and counted in a haemocytometer.

ADMINISTRATION OF IL-1ra

Two separate experiments using IL-1 ra were completed. In both studies, human IL-1 ra $4 \mathrm{mg} / \mathrm{kg}$ body weight (Synergen) was administered subcutaneously to nine rabbits every six hours for a three day period. A group of eight control rabbits received subcutaneous injections of equal volumes of vehicle $(10 \mathrm{mmol} / \mathrm{l}$ citrate buffer, $140 \mathrm{mmol} / \mathrm{l} \mathrm{NaCl}, 0.5 \mathrm{mmol} / \mathrm{l}$ EDTA, $\mathrm{pH} \mathrm{6.5)} \mathrm{at} \mathrm{the} \mathrm{same} \mathrm{intervals.} \mathrm{All} \mathrm{animals} \mathrm{had}$ had arthritis induced 14 days earlier and were killed at day 17, six hours after the last dose of IL-1 ra.

HISTOLOGICAL EXAMINATION OF SYNOVIAL LINING

The infrapatellar fat pad was dissected from both the arthritic and control knee joints, cut into two and fixed in formol buffered saline and paraffin embedded. Sections were cut at $5 \mu \mathrm{m}$, stained with haematoxylin and eosin, and examined microscopically.

QUANTITATIVE CYTOCHEMICAL ASSAY OF SYNOVIAL COLLAGEN CONTENT

Serial sections $5 \mu \mathrm{m}$ thick were cut from paraffinembedded blocks of control and arthritic infrapatellar fat pads. One section was stained with haematoxylin and eosin for evaluation of synovial histology. The other sections were dewaxed, hydrated and stained with $0 \cdot 1 \%$ sirius red F3BA (a stoichiometric stain for collagen ${ }^{11}$ ) in saturated aqueous picric acid for 30 minutes as described elsewhere. ${ }^{12}$ They were then dehydrated, cleared, and mounted in DPX. A Vickers M85a scanning and integrating microdensitometer was used to quantify the collagen content in the sections of synovial lining. ${ }^{6} 13$ The microdensitometer settings were: objective magnification $\times 25$; wavelength $530 \mathrm{~nm}$; spot size 2 ; mask size 2 ; scan speed 1 .

ASSESSMENT OF CARTILAGE METABOLISM

The rate of loss of proteoglycan from arthritic joints was indirectly estimated by measuring the glycosaminoglycan (GAG) content of the joint wash. Aliquots of joint wash were digested with papain at $60^{\circ} \mathrm{C}$ for 18 hours and the concentration of sulphated GAGs measured by an automated modification ${ }^{14}$ of the 1,9-dimethylmethylene blue binding assay. ${ }^{15}$ In addition, full thickness slices of articular cartilage were dissected from the femoral condyle and tibial plateau of arthritic and control joints. The cartilage was blotted dry, weighed and digested with papain for 18 hours at $60^{\circ} \mathrm{C}$ and the concentration of sulphated GAGs determined as above. The hydroxyproline concentration of the digested cartilage was also determined, ${ }^{16}$ to provide a measure of the collagen content of the tissue, against which the changes in GAG concentration could be compared.

The synthesis of proteoglycan by rabbit articular cartilage was measured in one experiment by incubating fragments of femoral or tibial cartilage (in triplicate) in Dulbecco's modified Eagle's medium (DMEM) buffered with hydroxyethylpiperazine-ethanesulphonic acid (HEPES) and containing $100 \mu \mathrm{Ci}$ $\left(3.7 \times 10^{5} \mathrm{~Bq} / \mathrm{ml}\right){ }^{35} \mathrm{SO}_{4}$ (Amersham, England) for four hours at $37^{\circ} \mathrm{C}$ in $5 \%$ carbon dioxide in air. The labelled medium was removed and the cartilage extracted with $4 \mathrm{~mol} / \mathrm{l}$ guanidinium chloride plus $2 \%$ 3-[-(3-cholamidopropyl)-dimethylammonio]-1-propane sulphonate (CHAPS) and protease inhibitors $(0.1 \mathrm{~mol} / 1$ 6-aminohexanoic acid, $0.01 \mathrm{~mol} / \mathrm{l}$ disodium EDTA, $0.005 \mathrm{~mol} / \mathrm{l}$ benzamidine hydrochloride, $0.001 \mathrm{~mol} / \mathrm{h}$-ethylmaleimide and $0.005 \mathrm{~mol} / \mathrm{l}$ iodoacetamide), twice for 24 hours at $4^{\circ} \mathrm{C}$ and the residue was digested with papain for 18 hours at $60^{\circ} \mathrm{C}$. The concentrations of sulphated GAGs in the medium, extract, and residue were determined. The various fractions were pooled and chromatographed on Sephadex G-25, eluted with $2 \mathrm{~mol} / 1$ guanidinium chloride in 50 


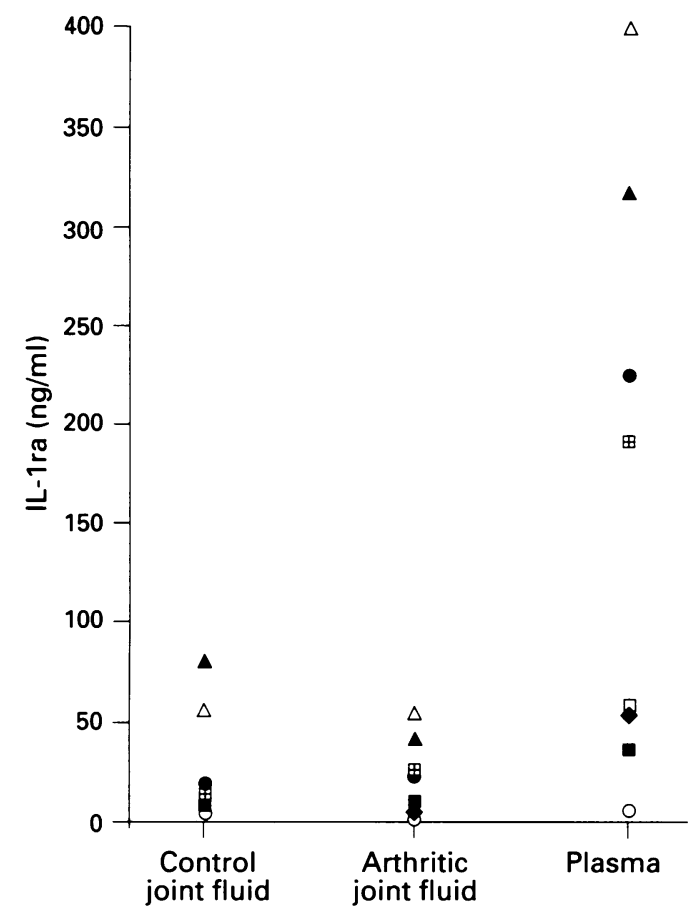

Figure 1 Concentrations of immunoreactive IL-1ra in the joint fluids of control and arthritic joints and in the plasma six hours after the last dose of this natural antagonist was administered. Results taken from both experiments, each animal denoted by a different symbol. Concentrations of immunoreactive IL-1 ra in animals not receiving human IL-1 ra were at the limit of detection.

$\mathrm{mmol} / 1$ sodium acetate, $\mathrm{pH} 5 \cdot 8$, plus $0 \cdot 1 \%$ Triton X-100 to remove unincorporated isotope; radioactivity in the void volume fractions was measured by scintillation spectrometry. Results were again expressed in relation to the collagen (hydroxyproline) content of the tissue.

ASSAY OF IL-1ra

Human IL-1ra was assayed in the plasma and synovial joint washes by use of a commerical

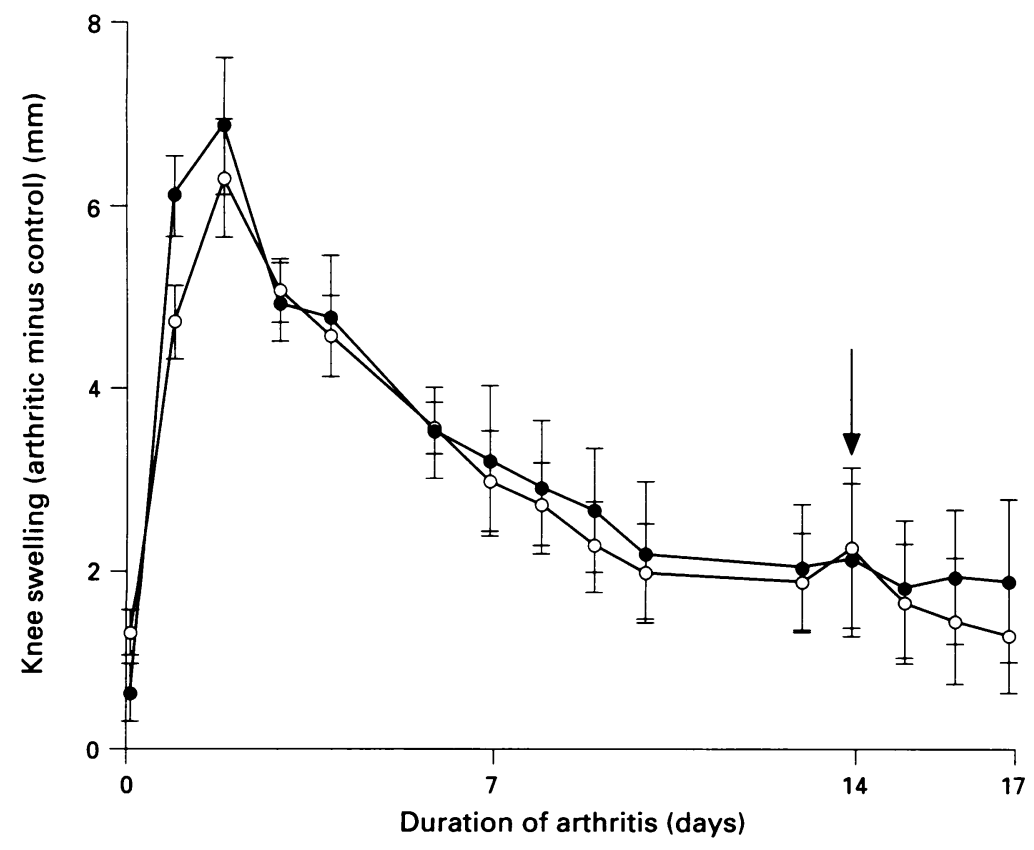

Figure 2 foint swelling in vehicle (O) and IL-1 ra (O) treated rabbits over the 17 day period of the experiment. Arrow indicates commencement of administration of IL-1ra or vehicle. Results are mean and SEM for groups of five or six rabbits. immunoassay ( $R$ \& D Systems, Oxford, England) used according to the manufacturer's instructions.

STATISTICAL ANALYSIS

The data were examined by use of the $\mathrm{N}$-score transformation method and shown to be normally distributed, therefore the statistical significance of the results was determined by Student's $t$ test.

\section{Results}

IL-1 ra CONCENTRATIONS IN JOINT WASHES AND PLASMA

It was possible to detect this natural antagonist in plasma and joint washes taken six hours after the last administration of IL-1 ra. The circulating concentrations of IL-1 ra were 33 (SEM 15) $\mathrm{ng} / \mathrm{ml}(\mathrm{n}=3)$ in experiment 1 and $237(59) \mathrm{ng} / \mathrm{ml}(\mathrm{n}=5)$ in experiment 2 . The concentrations of IL-1 ra in control and arthritic joints were similar in both experiments: $8(1 \cdot 1) \mathrm{ng} / \mathrm{ml}(\mathrm{n}=3)$ in arthritic joints and $7.5(2 \cdot 5) \mathrm{ng} / \mathrm{ml}(\mathrm{n}=3)$ in control joints in experiment 1 , and 36 (14) $\mathrm{ng} / \mathrm{ml}$ and $36(7 \cdot 3) \mathrm{ng} / \mathrm{ml}$, respectively, in experiment 2 (fig 1). The concentrations of IL-1 ra in joint fluids and plasma of rabbits not receiving exogenous IL-1ra were very low-at or below the limit of detection of the assay: the commercial antibody failed to recognise rabbit IL-1 ra at a concentration of $1 \mathrm{mg} / \mathrm{ml}$. Recovery of human IL-1 added to rabbit joint washes was between 84 and $133 \%$.

\section{EFFECT OF IL-1 1 ra ON JOINT SWELIING}

As normal after induction of arthritis, there was rapid swelling of the inflamed joints which reached a maximum between one and two days. Swelling then declined over the next seven days to reach a plateau of 2-3 $\mathrm{mm}$, which was then maintained for the duration of the arthritis. In both experiments the administration of IL-1ra at day 14 of the arthritis had no effect on the chronic joint swelling. Figure 2 shows the results from one experiment.

\section{EFFECT OF IL-1 ra ON LEUCOCYTE}

ACCUMULATION

At 17 days after induction of arthritis, large numbers of leucocytes were found in the arthritic joint washes. In contrast, the contralateral control joints contained no measurable leucocytes. The administration of IL-1ra from day 14 to day 17 resulted in a $60 \%$ decrease in the numbers of leucocytes in the inflamed joint cavity, but because of the variation in the leucocyte counts this decrease was not statistically significant. Figure 3 shows the combined results from the two experiments.

EFFECT OF IL-1 ra ON SYNOVIAL

HISTOPATHOLOGY

Contralateral control joints demonstrated a normal histological appearance; in contrast, 


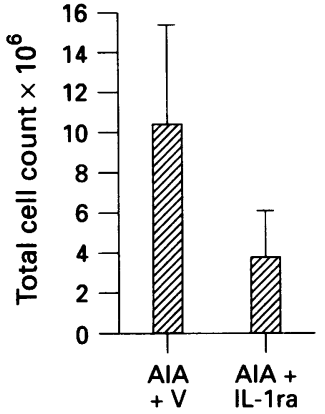

Figure 3 Numbers of leucocytes in the inflamed joints of the vehicle $(V)$ and IL-1 ra treated rabbits killed six hours after the last administration of IL-1ra. Results are mean and SEM for eight or nine rabbits and are the combined results from experiments 1 and 2. The difference is not statistically significant. $A L A=$ Antigen induced arthritis. inflamed joints showed an extensive mononuclear cell infiltrate, thickening of the synovial lining cell layer, increased vascularity, and increased connective tissue matrix content. The administration of IL-1 ra did not significantly reduce the cellularity of the synovium, but did cause a decreased extracellular matrix staining.

EFFECT OF IL-1 ra ON SYNOVIAL LINING

\section{COLLAGEN CONTENT}

The synovial lining of the infrapatellar fat pad of the control joints was mainly of the adipose type of subintima, with thin bands of collagen running through the tissue as identified by picrosirius red staining (fig 4A). In contrast, after 17 days development of arthritis the inflamed joints showed intense collagen staining throughout the matrix (fig $4 \mathrm{~B}$ ), confirming the findings of earlier investigations. ${ }^{6}$ The many fat spaces in the subintima had
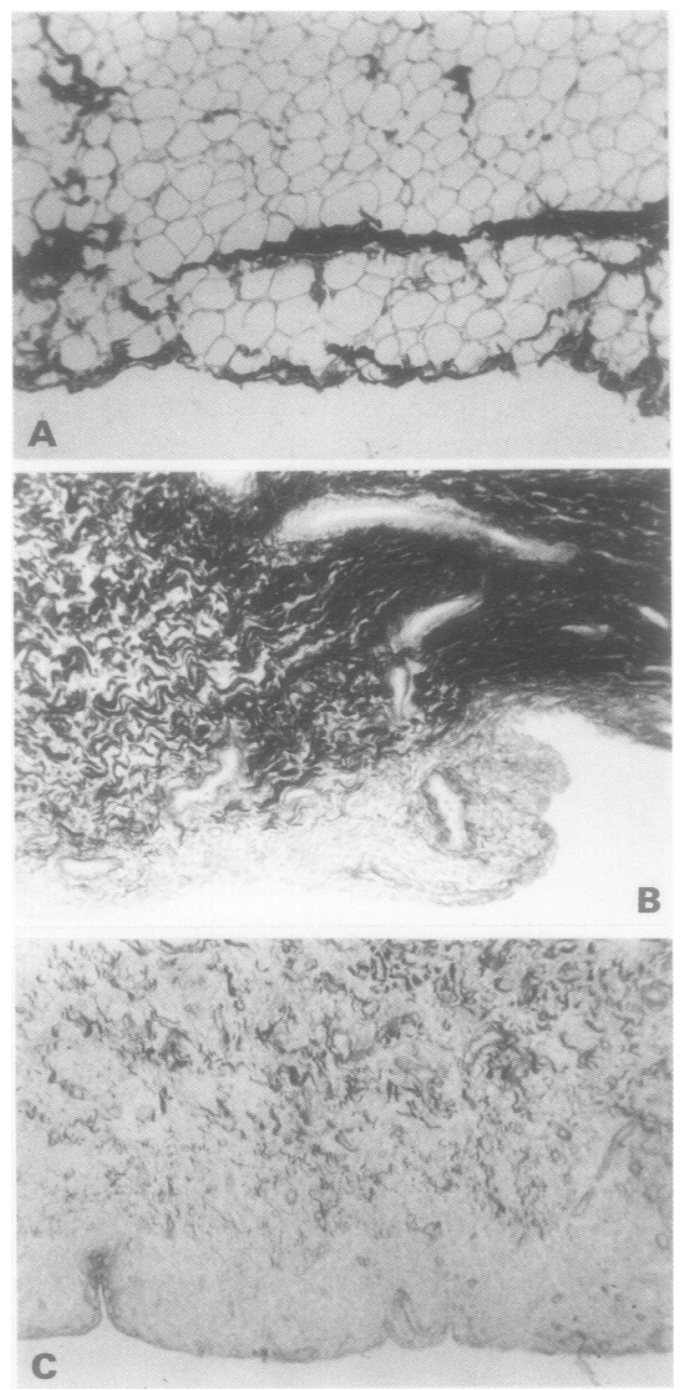

Figure 4 Distribution of collagen in sections of synovial lining as detected by use of the stoichiometric collagen stain, picrosirius red SR. A: Synovial lining from a control joint, showing a mainly adipose type subintimal tissue with occasional thin bands of collagen. B: Synovial lining from the inflamed joint of a rabbit administered vehicle, showing intense staining for collagen throughout the tissue. C: Arthritic synovium from a rabbit administered IL-1 ra, showing greatly reduced intensity of collagen staining.

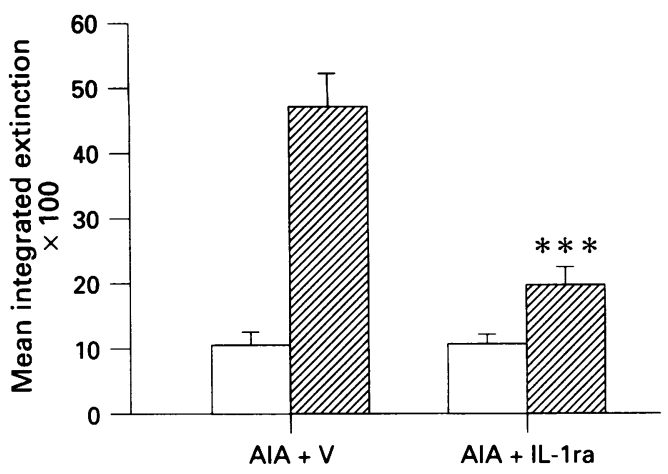

Figure 5 Effect of IL-1ra on the collagen content in the synovial lining of control $(\square)$ and inflamed $(\square)$ joints of rabbits treated with vehicle $(V)$ or with IL-1 ra, as measured by microdensitometric measurement of picrosirius red stained sections. Results are combined from two experiments and represent the mean and SEM for nine rabbits. $\star \star * ⿻<00.001 . A I A=$ Antigen induced arthritis.

disappeared and were replaced by collagenous matrix. Administration of IL-1 ra was associated with a noticeable decrease in collagen staining and, in six of eight animals, with the reappearance of subintimal fat spaces (fig 4C). Microdensitometric measurement of picrosirius red stain in sections revealed that the administration of $\mathrm{IL}-1$ ra produced a $65 \%$ decrease in the synovial lining collagen content. Figure 5 shows the pooled results from both experiments.

EFFECT OF IL-1 ra ON ARTICULAR CARTILAGE PROTEOGLYCAN CONTENT

In confirmation of the findings of previous studies, ${ }^{17}$ there was a significant loss of proteoglycan from both the femoral (mean of 35\%) and tibial (mean 28\%) articular cartilages of arthritic joints compared with contralateral control joints in animals killed 17 days after induction of arthritis. As predicted from a previous study, ${ }^{17}$ there was no increase in the concentration of proteoglycan fragments found in the arthritic joint fluids during this chronic phase of the arthritis. Administration of IL-1 ra from day 14 to day 17 had no influence on the loss of articular cartilage proteoglycan content (fig 6).

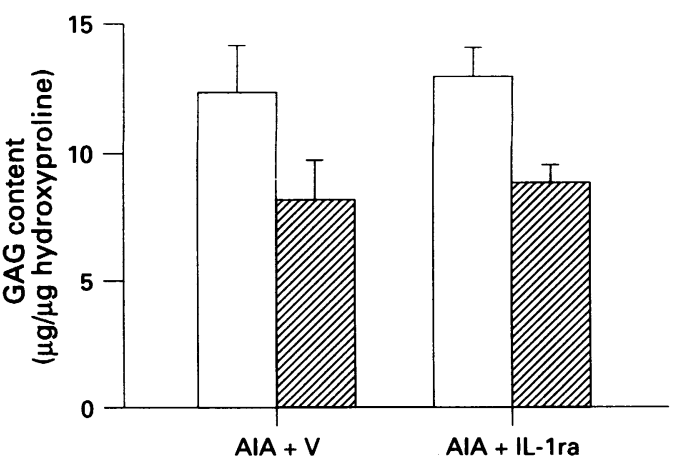

Figure 6 Effect of IL-1 ra on the proteoglycan (glycosaminoglycan, $G A G$ ) content of femoral cartilage from control $(\square)$ or arthritic $(\bigotimes)$ joints from rabbits treated with vehicle $(V)$ or IL-1 ra. Results are mean and $S E M$ for five or six rabbits. $A L A=$ Antigen induced arthritis. 


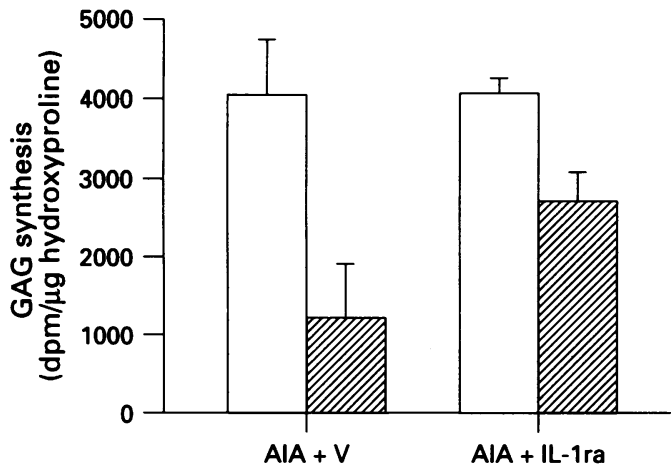

Figure 7 Effect of IL-1ra on proteoglycan synthesis

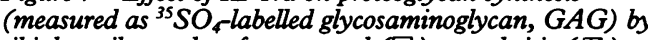
tibial cartilage taken from control (D) or arthritic (『) joints of animals treated with vehicle $(V)$ or IL-1 ra. Results are mean and SEM of five or six rabbits. ALA = Antigen induced arthritis.

EFFECT OF IL-1ra ON CARTILAGE

PROTEOGLYCAN SYNTHESIS

In one experiment, the influence of IL-1 ra on cartilage proteoglycan synthesis was determined. In vehicle treated rabbits there was a $68.5(12) \%$ inhibition of proteoglycan synthesis in inflamed joints compared with contralateral joints. In rabbits treated with IL-1ra, this decrease in proteoglycan synthesis was only 31.3 (11)\% (fig 7). This partial relief of proteoglycan synthesis was, however, not statistically significant.

\section{Discussion}

There is strong indirect and direct evidence for the hypothesis that IL-1 is a major mediator of the inflammation and tissue destruction seen in the rheumatoid joint. ${ }^{7}$ Injection of IL-1 into rabbit knee joints induces both a synovitis and the loss of proteoglycan from articular cartilage, ${ }^{18}$ and this effect can be blocked by IL-1 ra. ${ }^{9}$ Antigen induced arthritis in the rabbit is the only model of rheumatoid arthritis which is as chronic as the natural disease, lasting a substantial fraction of the lifespan of the animal, much in the way that the natural disease does. Arthritis begins with an acute synovitis which undergoes a transition to a chronic inflammatory and destructive lesion with pannus formation. The chronic phase of antigen induced arthritis is characterised by extensive cellular proliferation and fibrosis of the synovial lining, in association with progressive degradation of the articular cartilage..$^{2-6}$ IL-1 is detectable in the joint wash in the very early disease and is thereafter undetectable. However, the capacity of the inflamed synovial lining to synthesise bioactive IL-1 increases with increasing duration of the synovitis. ${ }^{19}$ It was therefore surprising that the administration of IL-1 ra to rabbits over the first three days of the induction of arthritis had no effect on either the synovitis or cartilage proteoglycan loss. ${ }^{10}$

In this study we have addressed the question of the effect of administering IL-1 ra to rabbits with established disease. At the time chosen for administration of the IL-1ra (day 14), the synovial lining contains a chronic inflam- matory infiltrate, there is substantial cellular proliferation, and this tissue is showing clear signs of fibrosis. ${ }^{6}$ In addition, there is substantial loss of cartilage proteoglycan at this time.

Immunoreactive IL-1 ra was detected in both the joint wash fluid and the plasma in samples taken six hours after the final administration of this recombinant protein. The initial half life of IL-1 ra in man has been estimated to be 21 minutes and the terminal half life 108 minutes. ${ }^{20}$ As IL-1 ra was administered every six hours, the concentrations measured would represent the smallest concentrations found in the body fluids during the course of treatment. The concentrations of $\mathrm{IL}-1 \mathrm{ra}$ in plasma were in the range $6-400 \mathrm{ng} / \mathrm{ml}$ and those in the second experiment were 10 -fold greater than in the first experiment; it is not clear why there was this difference in recorded concentrations between the two experiments. These experiments were done some six months apart, and there may have been some denaturation of the IL-1 ra in the synovial wash from the first experiment during storage, resulting in a loss of immunoreactivity and assay signal. However, the key finding from these measurements is that, taking into account the dilution effect of the joint wash (which is obtained by injecting $1.5 \mathrm{ml}$ of fluid into a cavity estimated to contain 100-200 $\mu \mathrm{l}$ of fluid ${ }^{21}$ and therefore calculated to be approximately 10 -fold), there was free access of the IL-1ra into the chronically inflamed joints in both experiments. Thus any failure to find positive effects cannot be attributable to failure to reach the site of tissue pathology. It should be noted that the commercial antibody used does not cross-react with IL-1 or with rabbit IL-1 ra.

Administration of IL-lra had no influence on joint swelling. There was a trend suggesting this molecule was having some effect on leucocyte accumulation, but this did not reach statistical significance. IL-1 ra did not inhibit the cartilage proteoglycan loss. There was some effect on the inhibition of cartilage proteoglycan synthesis found in inflamed joints, but this did not reach statistical significance. In spite of the failure of IL-1 ra to influence these major disease parameters, there was a marked and statistically significant inhibition of the collagen content of the inflamed synovial lining which was clearly seen visually and quantitated by use of the stoichiometric stain picrosirius red. This was a striking finding and suggests that the IL-1ra not only causes an inhibition of collagen deposition, through inhibition of the ability of locally produced IL-1 to stimulate collagen synthesis by synovial fibroblasts, but may also stimulate resorption of collagen already laid down. The activity of IL-1 ra is ascribed purely to its ability to bind to the IL-1 receptors and no additional activities (for example protease activity) for this protein have been described. ${ }^{22}$ It is therefore unlikely that IL-1 ra can itself stimulate collagen breakdown. The possibility must be considered that, in neutralising IL-1, the IL-1 ra is inducing some other resorptive pathway. 
The inhibition of IL-1 mediated activity by IL-1 ra requires a molar excess of the latter, normally in the order of $10-100$-fold. ${ }^{7}$ The amount of $\mathrm{IL}-1$ administered in this study was equivalent to that used by Cominelli $e t a l^{23}$ who reported that this antagonist significantly suppressed leucocyte accumulation, tissue oedema, and tissue necrosis in experimental immune colitis in the rabbit.

IL-1 ra has recently been shown to have an antifibrotic effect on bleomycin or silica induced lung fibrosis in mice, and was able to prevent the formation of pulmonary fibrosis when administered just before intratracheal administration of bleomycin or silica, and to reduce existing lung fibrosis previously induced by these agents. ${ }^{24}$ Inhibition of fibrosis was not accompanied by an inhibition of leucocyte infiltration, which is in agreement with our findings. The mechanism of the antifibrotic effect of IL-1 ra was not investigated in these murine lung fibrosis models. In a separate study in which we administered neutralising antibodies to rabbit tumour necrosis factor $\alpha$ (TNF $\alpha$ ) for five days between day 16 and day 20 after induction of arthritis, there was no evidence of a reduction of fibrosis in the synovium. This was in spite of a significant anti-inflammatory effect of this antibody in the early stage of arthritis. ${ }^{25}$ Piguet et al have shown that $\mathrm{TNF} \alpha$ does play a role in bleomycin induced fibrosis, ${ }^{26}$ and the possibility exists that different cytokine networks are at work in the lung and joint with respect to fibrosis.

In conclusion, administration of IL-1 ra to rabbits with established antigen induced arthritis had little effect on signs of inflammation or on cartilage proteoglycan loss. In contrast, the administration of this natural antagonist was accompanied by a rapid and profound antifibrotic response. These results, and the finding that IL-1 ra can inhibit experimentally induced lung fibrosis, argue for the therapeutic application of IL-1 ra as an antifibrotic agent.

We wish to thank the Arthritis and Rheumatism Council for Research and Synergen Inc for financial support. We are grateful to Dr Masura Yoshinaga, Kumamoto School of Medicine, Kumamoto 860, Japan for the provision of recombinant IL-1ra.

1 Henderson B, Edwards J C W. The synovial lining: in health and disease. London: Chapman and Hall, 1987.

2 Henderson B, Glynn L E, Chayen J. Cell division in the synovial lining in experimental allergic arthritis: proliferation of cells during the development of chronic arthritis. Ann Rheum Dis 1982; 41: 219-24.

3 Edwards J C W, Read N, Trefty B, Coulstock J, Henderson B. Quantitative histological analysis of antigen-induced arthritis in the rabbit. Br $\mathcal{f}$ Exp Pathol 1988; 69: 739-48.
4 Chew M W K, Henderson B, Edwards J C W. Antigeninduced arthritis in the rabbit: Ultrastructural changes at the chondrosynovial junction. Int $f$ Exp Pathol 1990; 71: 879-94.

5 Henderson B, Glynn L E, Bitensky L, Chayen J. Evidence for cell division in synoviocytes in acutely inflamed rabbit joints. Ann Rheum Dis 1981; 40: 177-81.

6 Beesley J E, Jessup E, Pettipher R, Henderson B. Microbiochemical analysis of changes in proteoglycan and collagen in joint tissues during the development of collagen in joint tissues during the development of
antigen-induced arthritis in the rabbit. Matrix 1992; 12: antigen-ind $189-96$.

7 Arend W P, Dayer J-M. Cytokines and cytokine inhibitors or antagonists in rheumatoid arthritis. Arthritis Rheum 1990; 33: 305-15.

8 Henderson B, Poole S. Modulation of cytokine function: therapeutic applications. Adv Pharmacol 1994; 25 53-115.

9 Henderson B, Thompson R C, Hardingham T, Lewthwaite J C. Inhibition of interleukin-1 induced synovitis and articular cartilage proteoglycan loss in the rabbit knee by recombinant human interleukin-1 receptor antagonist. Cytokine 1991; 3: 246-9.

10 Lewthwaite J, Blake S M, Hardingham T E, Warden P J, Henderson B. The effect of recombinant human interleukin-1 receptor antagonist on the induction phase interleukin-1 receptor antagonist on the induction phase
of antigen-induced arthritis in the rabbit. $\mathcal{F}$ Rheumatol of antigen-induced $1994 ; 21: 467-72$.

11 Junqueira L C U, Bignolas G, Brentani R R. A simple and sensitive method for the quantitative estimation of collagen. Anal Biochem 1994; 94: 96-9.

12 Sweat F, Puchtler H, Rosenthal S L. Sirius red F3BA as a stain for connective tissue. Arch Pathol 1964; 78: 69-72.

13 Henderson B. The application of quantitative cytochemistry to the study of diseases of the connective tissues. Progr Histochem Cytochem 1983; 15: 1-86.

Histochem Cytochem 1983; 15: 1-86.
14 Ratcliffe A, Doherty M, Maini R N, Hardingham T E. Increased concentration of proteoglycan components in the synovial fluid of patients with acute but not chronic joint disease. Ann Rheum Dis 1988; 47: 826-32.

15 Farndale R W, Buttle D J, Barrett A J. Improved quantitation and discrimination of sulphated glycosaminoglycans by use of dimethylmethylene blue. Biochim Biophys Acta 1986; 883: 173-7.

16 Stegemann $\mathrm{H}$, Stalder K. Determination of hydroxyproline. Clin Chim Acta 1967; 18: 267-73.

17 Pettipher E R, Henderson B, Hardingham T, Ratcliffe A. Cartilage proteoglycan depletion in acute and chronic antigen-induced arthritis. Arthritis Rheum 1989; 32: 601-7.

18 Pettipher E R, Higgs G A, Henderson B. Interleukin-1 induces leukocyte infiltration and cartilage proteoglycan degradation in the sync

19 Henderson B, Rowe F M, Bird C R, Gearing A J H. Production of interleukin-1 in the joint during the development of antigen-induced arthritis in the rabbit. Clin Exp Immuinol 1988; 74: 371-6.

20 Granowitz E V, Porat R, Mier J W, et al. Pharmacokinetics, safety and immunomodulatory effects of human recombinant interleukin-1 receptor antagonist in healthy humans. Cytokine 1992; 4: 353-60.

21 Delecrin J, Oka M, Kimar P, et al. Measurement of synovia fluid volume: A new dilution method adapted to fluid fluid volume: A new dilution method adapted to fluid 19: $1716-52$.

22 Arend W P. Interleukin-1 receptor antagonist: A new member of the interleukin-1 family. $\mathcal{F}$ Clin Invest 1990; 88 1445-51.

23 Cominelli F C, Nast C C, Clark B D, et al. Interleukin-1 (IL-1) gene expression, synthesis, and effects of IL-1 receptor blockade in rabbit immune complex colitis. f Clin Invest 1990; 86: 972-80.

24 Piguet P F, Vesin C, Grau G E, Thompson R C. Interleukin-1 receptor antagonist prevents or cures pulmonary fibrosis elicited in mice with bleomycin or silica. Cytokine 1993; 5: 57-61.

25 Lewthwaite J, Blake S, Hardingham T, et al. Role of TNFo in the induction of antigen induced arthritis in the rabbit in the induction of antigen induced arthritis in the rabbit and the anti-arthritic effect of species specific TNF $\alpha$
neutralising monoclonal antibodies. Ann Rheum Dis 1995; 54: $366-74$

26 Piguet P F, Collart M A, Grau G E, Kapanci Y, Vassalli P. Tumor necrosis factor/cachectin plays a key role in bleomycin-induced pneumopathy and fibrosis. $\mathcal{F} \operatorname{Exp} \mathrm{Med}$ 1989; 170: 655-63. 